\title{
Photosynthesis as a Discrete Biochemical Process
}

\author{
J. KREMPASKÝ* and M. ŠTETINA \\ Department of Physics, Faculty of Electrical Engineering and Information Technology, \\ Slovak Technical University, Ilkovičova 3, 81219 Bratislava, Slovakia
}

(Received 25 January 2000)

\begin{abstract}
It is shown that the existence of a multioscillatory and chaotic regime observed in the photosynthesis could be explained on the basis of logistic equations, i.e., using a discrete approach. Transforming known phenomenological differential equations describing the photosynthesis into the discrete formalism it is possible to demonstrate that by change of control parameters such equations generate the very well known Feigenbaum's scenario of the duplication of states including the possibility of the transition into a chaotic regime. The multioscillatory states characterised by even, and what is surprising, also by odd number of "subcycles" are generated at some special combinations of values of control parameters.
\end{abstract}

Keywords: Photosynthesis; Multioscillatory regime; Chaos; Feigenbaum's scenario

\section{INTRODUCTION}

The glycolysis and photosynthesis belong to the fundamental processes characterising living systems. The situation in the problems of glycolysis is optimistic one, because the focused theoretical and experimental research is now practically in the stage of a quantitative simulation, but the situation with the photosynthesis is much more complicated. The first information concerning the photosynthetic oscillations was done by Wilson and vin (Wilson and Calvin, 1955) and the first theoretical formulation of this problem appeared few years later in a paper by Chernavskaya and
Chernavskii (Chernavskaya and Chernavskii, 1960). However, this theory was not able to explain the large spectrum of pecularities observed in the photosynthesis.

Periodic processes in plants were experimentaly studied (Yamazaki et al., 1965) and the observed phenomena were interpreted (Degn and Mayer, 1969). The influence of some external factors on photosynthetic oscillations was also studied (Butler, 1957; Laisk, 1977).

It is possible to find a large amount of experimental data concerning the photosynthetic oscillations (Krempaský et al., 1993; Krempaský et al., 1995), where also a theoretical explanation

* Corresponding author. 
of the observed phenomena based on modified equations of Chernavskaya and Chernavskii was attempted.

A serious mathematical analysis of time series of signals registered in photosynthetic reactions (Bokes and Krempaský, 1997) shows that photosynthetic systems clearly exhibit the behaviour characteristic for pure oscillations, stochastic as well as deterministic chaos. A very probable genesis of the stochastic chaos is connected with bubbles generated by oxygen molecules arising in the process of photosynthesis. However, the origin of multioscillatory regimes and of the deterministic chaos remain unclear, because proposed mechanisms of the multiplication of frequences appear to be non realistic and for what concerns the deterministic choas it was not proven that the three evolution equations proposed really have chaotic solutions.

It seems that the problem of photosynthetic oscillations is fundamentally important, becauseas Walker (Walker, 1992) is quoting from the paper of Laisk (Laisk, 1989) - “... to understand oscillations is to understand photosynthesis". When we try to find the response to the principal question, what is really oscillating at the photosynthesis, we come into troubles, which Walker mentioned using the words: The short answer to "what oscillates" is "everything".

In principle there exist two possibilities: (a) the photosynthetic system works as a group of autonomous biochemical oscillators and the result is that "everything oscillates", (b) the photosynthetic system is internally corelated complex system, which by using a special (up to now not precisely known) mechanism generates a large variety of oscillations recorded by the measurement.

The first possibility seems to be supported by a very complex model elaborated by Laisk et al. (Laisk et al., 1989), based on concrete biochemical processes. This model contains no less than 17 evolution equations. The numerical solution to these equations offers a large amount of results, but gives only very little information concerning oscillations. Larger amount of information can be found in the paper of Laisk and all (Laisk et al., 1991), but this paper has nothing to do with the model mentioned above.

We would like to support in this paper the second possibility, i.e., the point of view that the photosynthetic system works as an integral unique system. It is not our aim to work out a new model based on a concrete mechanism, but we want to discover a possible mechanism which is responsible for generating of a large variety of frequencies found at the measurement. The equations formulated by Chernavskaya and Chernavskii will be a starting point of our calculations. One of the possible mechanisms of producing of photosynthetic oscillations we have found could be e.g., the Feigenbaum's scenario of the multiplication of states. We shall try to demonstrate that the adequate solution to the basic equations could lie in the discretness of the photosynthetic process. Due to the large biomolecules taking part in these processes the discretness of considered phonomena apears to be very probable.

\section{THEORY}

It was shown (see e.g., Rowlands, 1990; Feigenbaum, 1978; Buchanan and Turner, 1992) that logistic equations describing processes exhibit in general a broader spectrum of possibilities as differential equations. Chemical processes are always realised as discrete ones (there exist the interactions between atoms or molecules), therefore only the discrete formalism in describing of these processes is adequate. It is based on the known "occupying numbers" and "transite probabilities". The aim is to express the change of a characteristic quantity (e.g., the concentration defined as a mean value of the number of entities is a volume unit $\langle N\rangle)$ per time unit. This change can be expressed in two ways:

$$
\text { (a) } \frac{d\langle N\rangle}{d t}
$$


(for a continuous approach) resp.

$$
\text { (b) } \frac{\left[\langle N\rangle_{n+1}-\langle N\rangle_{n}\right]}{\varepsilon}
$$

(for a discrete approach) where $\langle N\rangle_{n+1}$ is the mean number of entities in $(n+1)$-th step, $\langle N\rangle_{n}$ the same in the $n$-th step and $\varepsilon$ is the duration of elementary processes. Two various ways of the description mentioned above can be demonstrated on a example of a simple autocatalytic reaction defined by the scheme:

$$
A+X \underset{k_{-}}{\stackrel{k_{+}}{\rightleftarrows}} 2 X
$$

The appropriate evolution equations for this reaction is of the form (see e.g., Haken, 1978):

$$
\begin{aligned}
\frac{d\langle N\rangle}{d t} & =\frac{\left[\langle N\rangle_{n+1}-\langle N\rangle_{n}\right]}{\varepsilon} \\
& =k_{+} N_{A}\langle N\rangle-k_{-}\langle N(N-1)\rangle
\end{aligned}
$$

where $k_{+}$and $k_{-}$are reaction constants and $N_{A}$ the concentration of the raw material. Supposing $N$ being large and denoting $\langle N\rangle=N$ we can transform the Eq. (4) into two versions:

$$
\frac{d N}{d t}=a N_{A} N-b N^{2}
$$

(for a continous approach) and

$$
N_{n+1}-N_{n}=\varepsilon\left[a N_{A} N_{n}-b N_{n}^{2}\right]
$$

(for a discrete approach).

It is seen that the general form of an evolution equation in the continous approach is of the form

$$
\frac{d y}{d t}=F(y, \lambda)
$$

where $y$ is a characteristic quantity and $\lambda$ a control parameter. Its analog in the discrete formalism is the logistic equation:

$$
y_{n+1}=G\left(y_{n}, \lambda\right)
$$

where $y_{n+1}$ and $y_{n}$ are values of the characteristic quantity in the $(n+1)$-th step. The transformation relations between these two equations are of the form

$$
y_{n+1}=y_{n}+\frac{d y_{n}}{d t} \varepsilon
$$

and

$$
\frac{d y}{d t}=\frac{1}{\varepsilon}\left(y_{n+1}-y_{n}\right)
$$

Using these transformations we can move from a continuous approach to a discrete one and vice versa.

It is known that nature is discrete in principle, therefore there exists only one adequate approach to its phenomena-the discrete formalism. However, the physical quantities are in many cases distributed into so small quanta that appropriate processes seem to be continous and therefore it is possible to use differential equations for their description. This statement is correct for atomic processes, but it could not be valid for processes in which heavy biomolecules participate. We suppose that the photosynthesis reallised by a dark Calvin's cycle could be the case, therefore we tried to analyse this phenomenon using the discrete approach.

We shall start with two fundamental evolution equations derived by Chernavskaya and Chernavskii, which have the form

$$
\frac{d X}{d t}=a_{1}+b_{1} X^{2}-c_{1} X Y
$$

$$
\frac{d Y}{d t}=a_{2} X^{2}-b_{2} X Y-c_{2} Y^{2}
$$

where $X$ and $Y$ are the concentrations of two dominante components (the triose and hexose). Using the transformation (10) it is easily possible to found their logistic equivalents

$$
\begin{array}{r}
X_{n+1}=A_{1}+X_{n}+B_{1} X_{n}^{2}-C_{1} X_{n} Y_{n} \\
Y_{n+1}=Y_{n}+A_{2} X_{n}^{2}-B_{2} X_{n} Y_{n}-C_{2} Y_{n}^{2}
\end{array}
$$


where $A_{1}=\varepsilon a_{1} ; B_{1}=\varepsilon b_{1} ; C_{1}=\varepsilon c_{1} ; A_{2}=\varepsilon a_{2}$; $B_{2}=\varepsilon b_{2} ; C_{2}=\varepsilon c_{2}$. The constants $A_{i}, B_{i}, C_{i}(i=1$; 2) are non-negative and we shall suppose in the computer analysis that $C_{1}=B_{2}=C$.

\section{RESULTS OF THE NUMERICAL ANALYSIS}

It is known that the chemical oscillations in some cases are stimulated by adding of some constituent into the system from the surroundings. On the basis of this knowledge we suppose that the fundamental control parameter in our case could be the flow defined by the constant $A_{1}$. After some thousands of numerical analysis we came to the conclusion that the comnbination of values of last constants in Eqs. (13) and (14) generating interesting results is the combination: $B_{1}=2.0$, $C_{1}=3.2, A_{2}=3.2, B_{2}=3.2$ and $C_{2}=1.8$. The value of the constant $A_{1}$ was changed in the

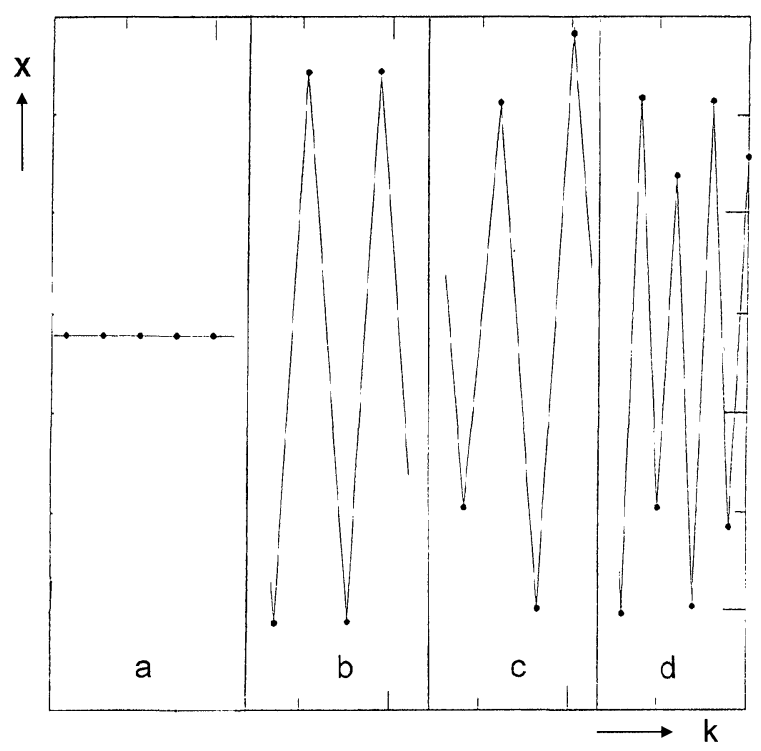

FIGURE 1 Time dependence of the quantity $X$ on the number of steps $\left(B_{1}=2.0, C_{1}=3.2, A_{2}=3.2, B_{2}=3.2, C_{2}=\right.$ 1.8, $X_{0}=0, Y_{0}=0.2$ ). (a) $A_{1}=0.02$, (b) $A_{1}=0.132$, (c) $A_{1}=0.2$, (d) $A_{1}=0.206$.

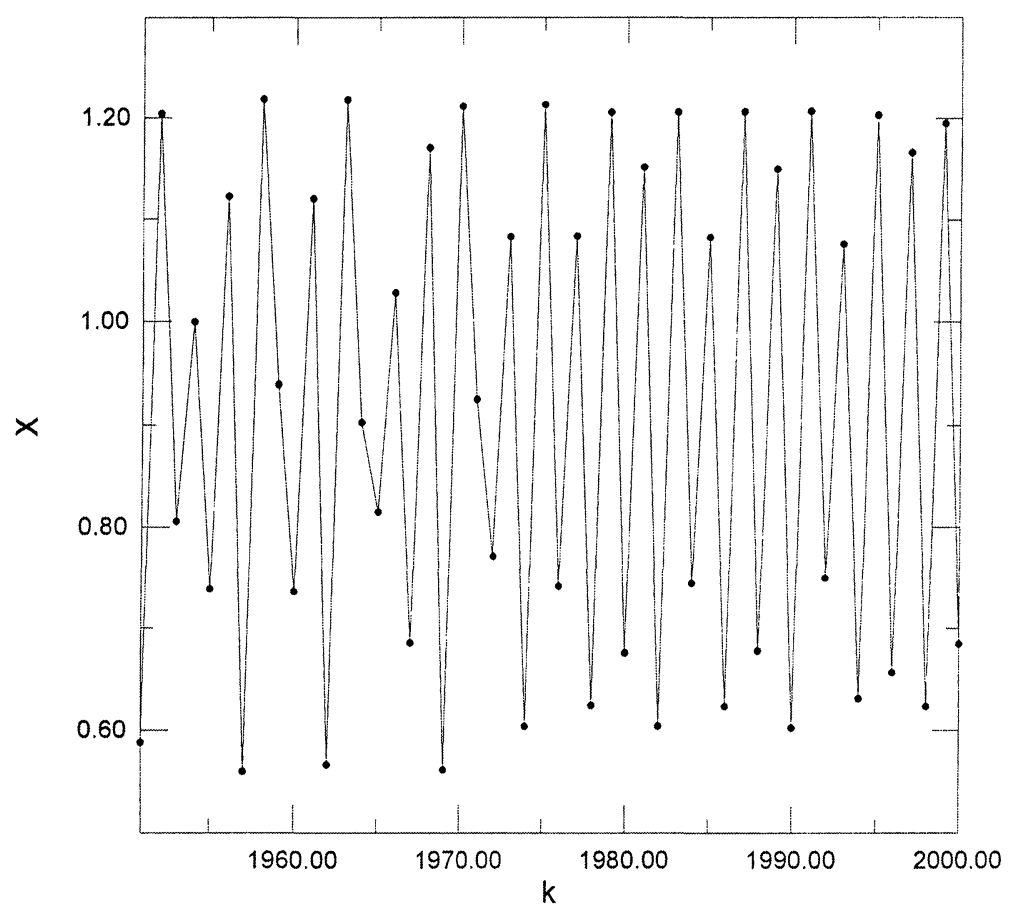

FIGURE 2 Time dependence of the quantity $X$ for last 50 steps belonging to the value $A_{1}=0.23$ demonstrating the deterministic chaos (last constants as in Fig. 1). 


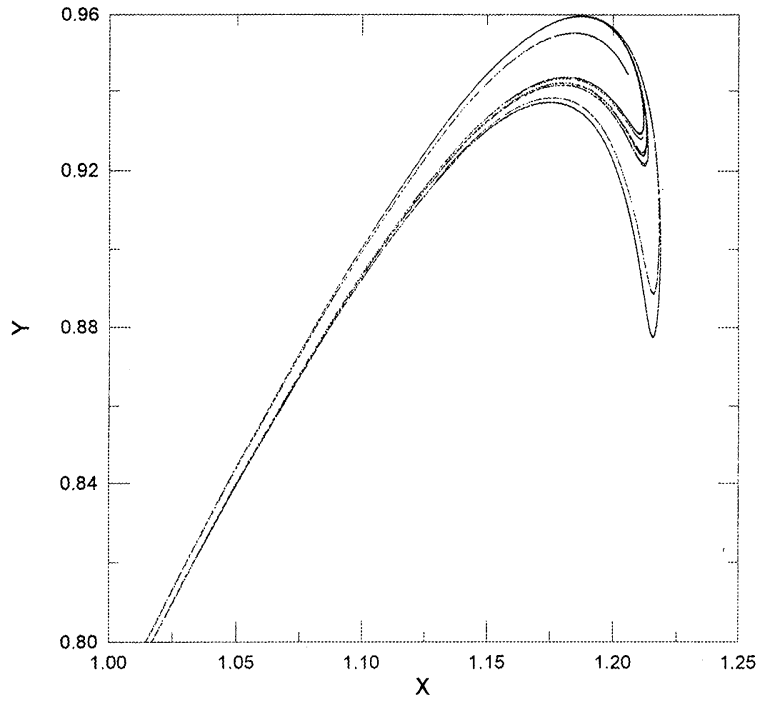

FIGURE 3 The fractal structure of the strange attractor belonging to the values given in Figure 5 .

interval $\langle 0 ; 0.231\rangle$ and the calculation was performed at the starting point $X_{0}=0$ and $Y_{0}=0.2$. Results of this calculation are demonstrated in Figures $1-4$. It follows from these results that the known Feigenbaum's scenario of the generation of the chaotic regime exhibiting the "duplication" of states is present in our system.

Only one stable state (Fig. 1a) exists in the interval $A_{1} \in\langle 0 ; 0.132\rangle$, in the interval $A_{1} \in\langle 0.132$; $0.184\rangle$ jumps between two possible states (oscillations) emerge (Fig. 1b), in the interval $\langle 0.184$; $0.205\rangle$ four possible states exist (Fig. 1c), in the interval $\langle 0.205 ; 0.211\rangle$ eight states (Fig. 1d), in the interval $\langle 0.211 ; 0.2111\rangle$ sixteen states etc. In the interval $A_{1} \in\langle 0.225 ; 0.231\rangle$ the regime of deterministic chaos takes place, which is documented in Figure 2. The phase portrait pictured in Figure 3 has a fractal stucture. The summary of all information mentioned above is represented by a bifurcation diagram in Figure 4 . It is worth noting that the numerical analysis of Eqs. (13) and (14) produces very interesting results also for $A_{1}>0.231$, but the quantities $X$ and $Y$ have in this region negative values, which is physically unrealistic.

In our system there exist some other interesting combinations of control parameters, which give new qualitative regimes. The phase diagram

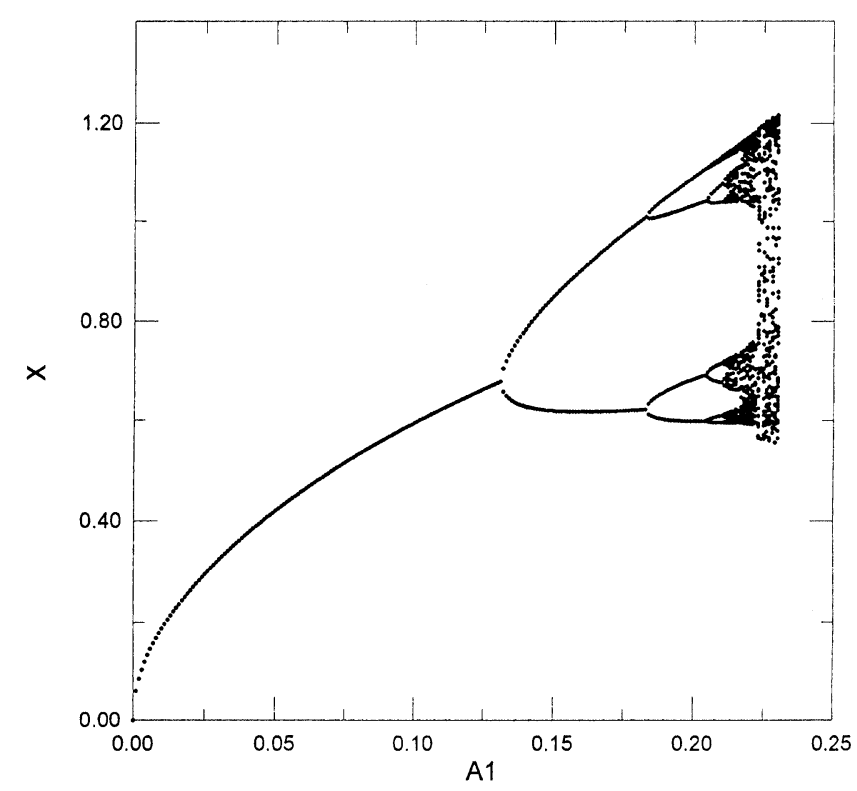

FIGURE 4 The bifurcation diagram of a photosynthetic system described by logistic equations. 
belonging to the combination: $A_{1}=0.21 ; B_{1}=0.6$; Figure 5. This diagram exhibits four "subcycles" $C_{1}=3.2 ; A_{2}=3.6 ; B_{2}=3.2$ and $C_{2}=1.2$ (for superposed on a basic cycle and represents the the same starting point as above) is shown in "multioscillatory" regime.

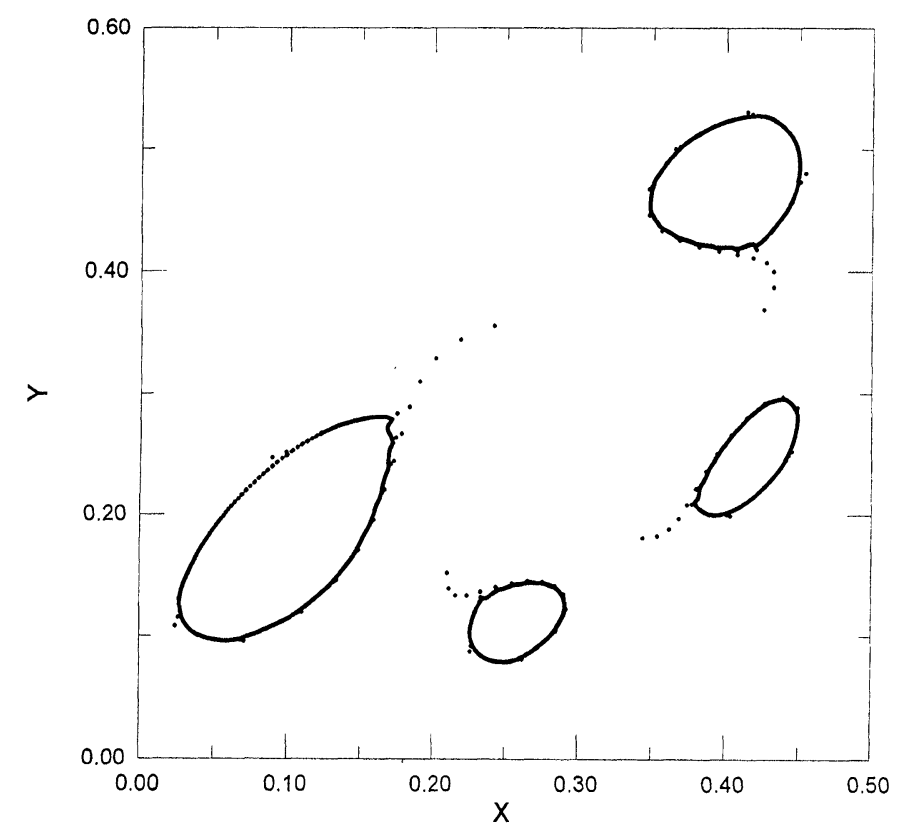

FIGURE 5 The phase portrait of a photosynthetic system belonging to the values: $A_{1}=0.21, B_{1}=0.6, C_{1}=3.2, A_{2}=3.6, B_{2}=3.2$, $C_{2}=1.2, X_{0}=0, Y_{0}=0.2$.

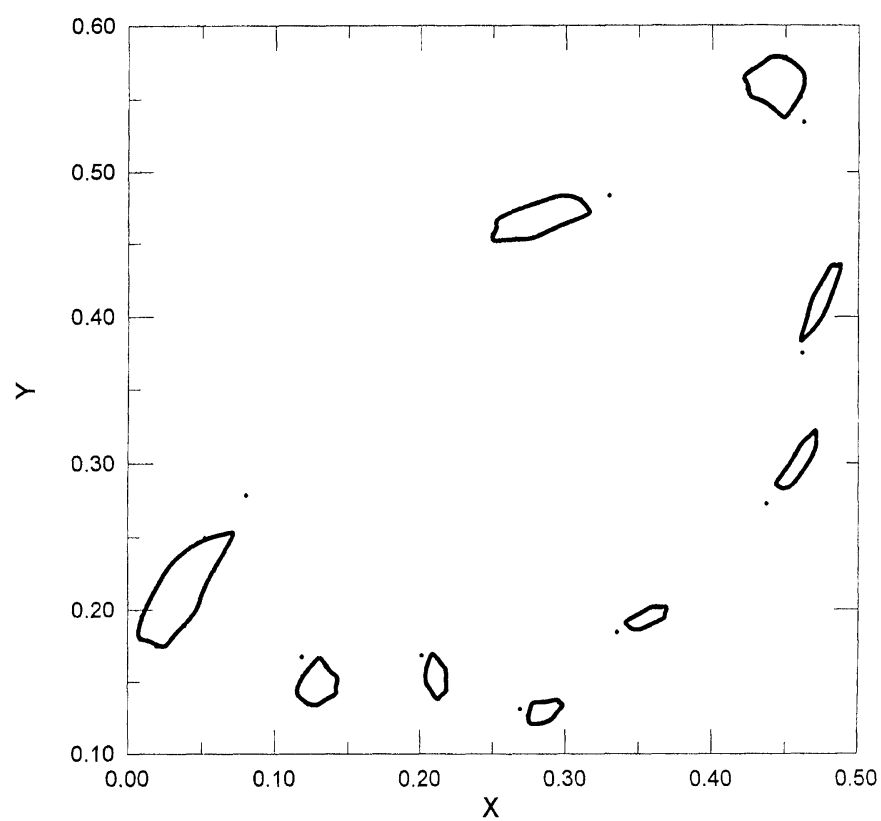

FIGURE 6 The phase portrait of a photosynthetic system belonging to the values: $A_{1}=0.201, B_{1}=1.2, C_{1}=3.4, A_{2}=3.8, B_{2}=3.4$, $C_{2}=0.8, X_{0}=0, Y_{0}=0.2$. 
A more complicated phase diagram is seen in Figure 6. This diagram belongs to the combination: $A_{1}=0.201 ; \quad B_{1}=1.2 ; \quad C_{1}=3.4 ; \quad A_{2}=3.8$; $B_{2}=3.4$ and $C_{2}=0.8$ and exhibits nine "subcycles". This is a surprising result, because the Feigenbaum's scenario is characterised only by odd (higher) number of states.

The results mentioned above certainly do not represent all possibilities generated by Eqs. (13) and (14), but it is clearly seen that a discrete approach produce much a richer spectrum of possibilities, than the continous one.

\section{DISCUSSION}

The experimental study of photosynthesis using the measurement of electromotive signals generated in this process proved the idea that this phenomenon is extremely complicated, variable and practicaly unreproducible. A simple model proposed by Chernavskaya and Chernavskii, 1960 and further elaborated by Krempaský et al., 1993, which is based on partial differential equations, was absolutely not able to interpret all the observed peculiarities.

The approach based on the use of more than two evolution differential equations was also not very successful, because it calculated with not very realistic mechanisms producing the multiplicity and chaos. It seems that a successful approach to the problems of photosynthesis consists in using the discrete formalism in the formulation and solving of basic equations.

We have shown that a discrete model of photosynthesis based only on two simple logistic equations is able to account for the experimentally measured data. The main result of this analysis is the finding that the process of photosynthesis depends very sensitively on the input of component into the system from the surroundings. A very small change in the value of this flow can produce a dramatic change in the state of the system. These changes can be generated during the measurement, therefore it is clear, why it is practically impossible to gain reproducible records. The presence of multioscillatory states and of the deterministic chaos under these circumstances is a very probable scenario.

From our analysis it follows that if we want to have reproducible results in the case of the photosynthesis, it is necessary to stabilise the conditions of the measurement, especially that of the constant $A_{1}$. This, however, seems to be a hard problem.

\section{References}

Bokes, P. and Krempaský, J. (1997) Chaos in photosynthesis: stochastic or deterministic? J. Electr. Engineering, 48, $312-315$.

Buchanan, J. L. and Turner, P. R. (1992) Numerical Methods and Analysis. McGraw-Hill, New York-St. Louis-San Francisco - Auckland - Bogota-Caracas-Lisbon-London - Madrid-Mexico-Milan-Montreal-New Delhi-ParisSan Juan-Singapore-Sydney-Tokyo-Toronto.

Butler, W. L. (1957) Transient phenomena in leaves as recorded by a gas thermal conductivity meter. In: Gaffron, H. (Ed.), Research in Photosynthesis, pp. 399-405. Natl. Acad. Sci. Natl. Research Council, New York-London.

Chernavskaya, N. M. and Chernavskii, D. S. (1960) Periodical effects in photosynthesis (in Russian). Uspechi fiz nauk, 72(3), $627-652$.

Degn, H. and Mayer, D. (1969) Theory of oscillations in peroxidase catalyzed oxidation reactions in open system. Biochim. Biophys. Acta, 180, 291-301.

Feigenbaum, M. J. (1978) Quantitative Universality for a class of Nonlinear Transformations. J. Stat. Phys., 19, 25-52.

Haken, H. (1978) Synergetics-An Introduction. SpringerVerlag, Berlin-Heidelberg-New York.

Krempaský, J., Smrčinová, M. and Ballo, P. (1993) Periodicity and chaos in a photosynthetic system. Photosynthesis Research, 37, 159-164.

Krempaský, J., Smrčinová, M. and Ballo, P. (1995) Oscillations in a Chloroplast Suspension System. Physiol. Biophys., 14, $463-471$.

Laisk, A. C. (1977) Kinetics of Photosynthesis and Photorespiration of $C_{3}$-Plants, pp. $72-131$. Nauka, Moskow (in Russian).

Laisk, A. C., Eichelmann, H., Oja, V., Eatherall, A. and Walker, D. A. (1989) A mathematical model of the carbon metabolism in photosynthesis. Difficulties in explaining oscillation by fructose 2,6 -bisphosphate regulation. Proc. R. Soc. Lond. B, 237, 389-415.

Laisk, A. C., Siebke, K., Gerst, U., Eichelmann, H., Oja, V. and Heber, U. (1991) Oscillations in photosynthesis are initiated and supported by imbalances in the supply of ATP and NADPH to the Calvin cycle. Planta, 185, 554-562.

Rowlands, G. (1990) Non-linear phenomena in Science and Engineering. Ellis Horwood, New York-Toronto-SydneyTokyo - Singapore.

Walker, D. A. (1992) Concerning oscillations. Photosynthesis Research, 34, 387-392.

Wilson, A. T. and Calvin, M. (1955) The photosynthetic cycle. $\mathrm{CO}_{2}$ dependent transients. J. Am. Chem. Soc., 77, $5948-5957$

Yamazaki, I., Yokota, K. and Nakajima, R. (1965) Oscillatory oxidations of reduced pyridine nucleotide by peroxidase. Biochem. Biophys. Res. Commun., 21, 582-586. 


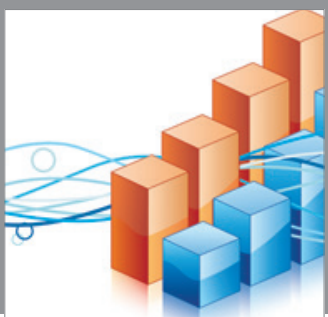

Advances in

Operations Research

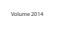

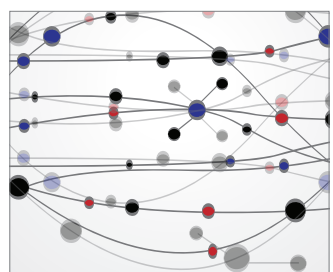

\section{The Scientific} World Journal
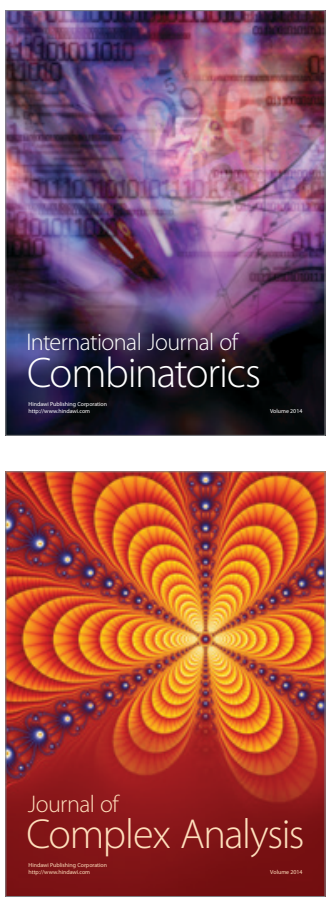

International Journal of

Mathematics and

Mathematical

Sciences
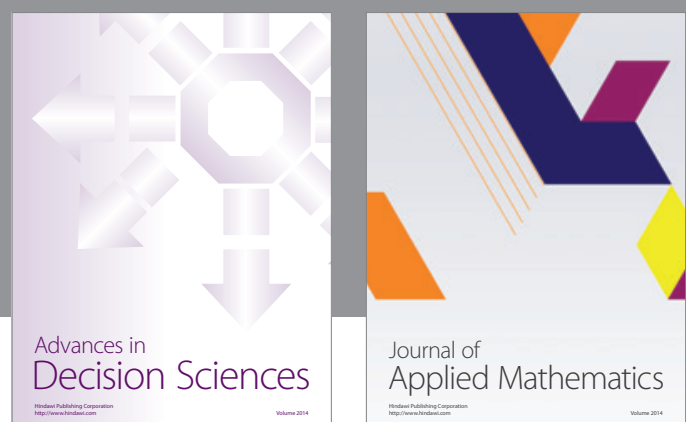

Journal of

Applied Mathematics
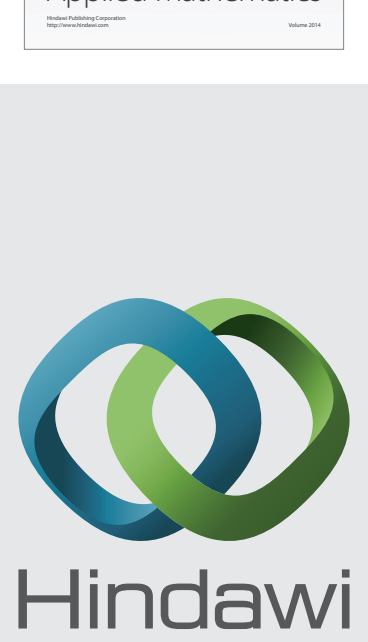

Submit your manuscripts at http://www.hindawi.com
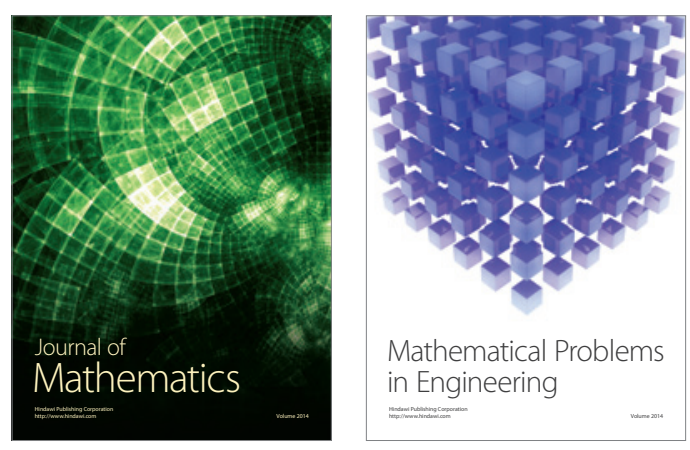

Mathematical Problems in Engineering
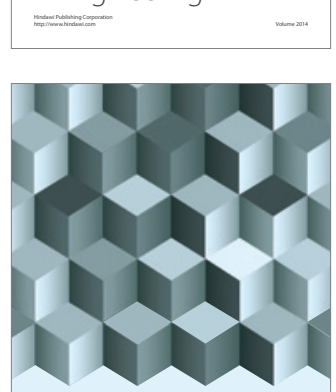

Journal of

Function Spaces
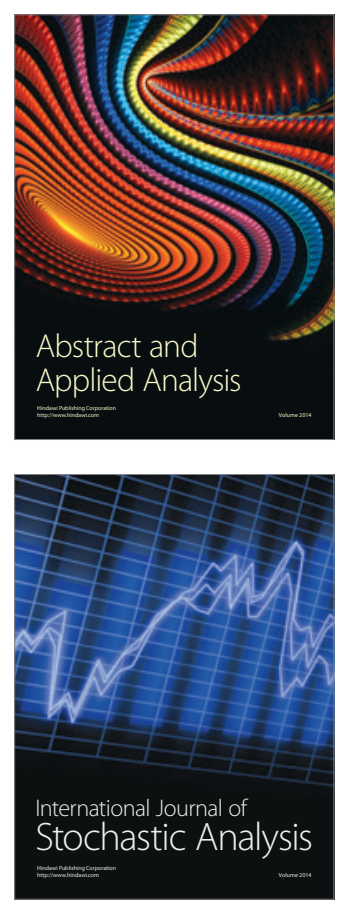

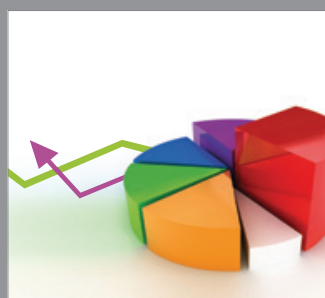

ournal of

Probability and Statistics

Promensencen
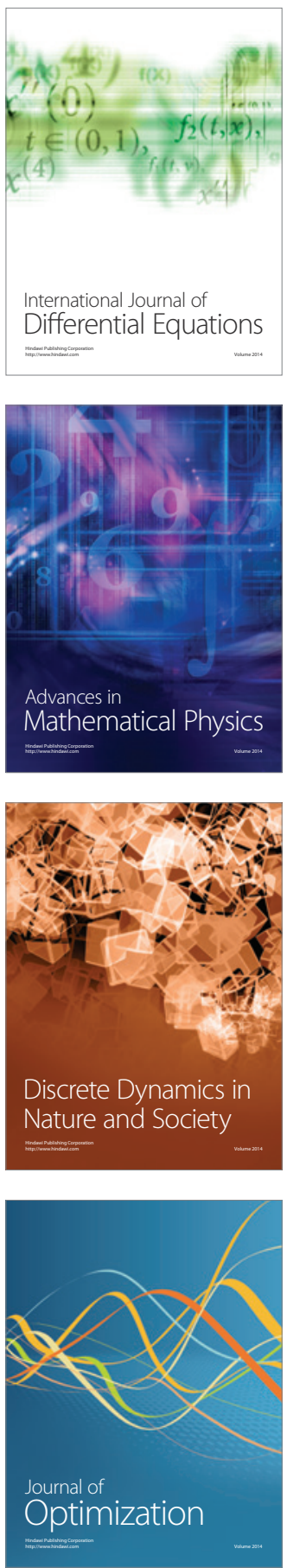